\title{
Societal Health and Improvement of Access to Care
}

\author{
Kelly L Colwell* and Karen H Larwin \\ Assistant Professor of Health Professions, USA
}

*Corresponding author: Kelly L Colwell, Assistant Professor of Health Professions, Program Director Respiratory Care Director, Master of Respiratory Care, USA

\section{ARTICLE INFO}

Received: 幽 July 12, 2019

Published: 蔧 July 23, 2019

Citation: Kelly L Colwell, Karen H Larwin. Societal Health and Improvement of Access to Care. Biomed J Sci \& Tech Res 19(5)-2019. BJSTR. MS.ID.003373.

\section{ABSTRACT}

Introduction: Maintaining and attending follow-up healthcare appointments, coupled with proper age- and disease-specific education, is paramount to preventing and managing asthma exacerbations, asthma flare-ups, hospitalizations, increased use of oral corticosteroids, and deaths from uncontrolled asthma symptoms. No show rates have an effect in the quality and management of chronic health conditions, limit access for those waiting to be diagnosed and begin treatment and creates a financial hardship for provider's practices like this, limiting the expansion of healthcare services in underserved areas. The focus of this study was to ascertain if age- and disease-specific education impact the no show rates or the healthcare phenomenon of the empty chair, for follow up healthcare management in the adolescent patient asthma populations.

Methods: A quasi-experimental, retrospective chart review was utilized for participant populations with a specific ICD-9/10 asthma diagnosis codes, ages 8-18 within Mahoning, Trumbull, Stark and Franklin Counties, Ohio. Demographic variables of age, gender, race, type of healthcare coverage, and geographic zone were compared to education received or not received. Appointment slot utilization variables of KEPT, no show, rescheduled and canceled was also collected. Pertinent data were then compiled with analysis performed by S.P.S.S statistical analysis software. Descriptive and inferential statistics were used to address all research questions.

Results: Analyzed data revealed the only correlation to the slot utilization variable, and education was KEPT. Geographic zone revealed that the highest KEPT appointments were in Mahoning County, highest no show rates were between the border of Trumbull/ Mahoning Counties. There was no significant correlation between no show rates and demographic variables.

Conclusion: Although education had an integral relationship with KEPT appointments, it was not inversely proportional to no show rates. Education encounters were related to the KEPT variable lending to an improvement in health literacy.

\section{Introduction}

Approximately 133 million U.S. citizens were afflicted with and suffering from at least one chronic health condition in 2005 (CDC, 2015). According to the CDC, it is approximated that by 2020 , more than 167 million individuals will be afflicted with and suffering from a single chronic illness. By the year 2020, it is estimated that individuals living with multiple chronic health conditions will meet, or surpass, 81 million [1,2]. The previous statistics represent many preventable diseases brought on by health determinants and individual unhealthy choices including certain cancers, diabetes, stroke, heart disease, and chronic pulmonary conditions, with pulmonary conditions as the number one reported chronic health condition in the U.S. (CDC, 2016c). Moreover, as of 2014, 86\% of total healthcare expenditures in the U.S. were directed towards individuals living with one or more chronic health diseases or conditions (Centers for Medicare and Medicaid Services, 2016a). Asthma is one chronic health condition in the U.S. that has afflicted over 25 million Americans, including 7 million children under the age of 18 (CDC, 2016c).

Asthma is considered the most common chronic disease in children for which there is no known cure, with $8.6 \%$ of children 
in the U.S. afflicted (U.S. Department of Health and Human Services, National Heart Lung and Blood Institutes, 2014b). Asthma accounts for most missed days of school, lost educational opportunities, missed days from work, and is responsible for over 439,000 hospitalizations, 14.2 million physician office visits, and 2 million emergency room visits, yearly (CDC, 2015). Moreover, there are 3,630 preventable deaths attributed, yearly, to poor asthma management, non-compliance with medications, and follow-up care, leading to a lack of asthma control of symptoms and resulting in 187 deaths, per year, in children (Asthma and Allergy Foundation of America [AAFA], 2015). Disparities in asthma mortality are noted, and death rates for asthma are $75 \%$ higher for ethnic minorities when compared with their White counterparts [3-7].

\section{Asthma Control}

The goal of asthma treatment is to control a patient's symptoms, prevent life-threatening exacerbations, and affect an improvement in pulmonary lung function resulting in a healthier, more productive quality of life. Asthma control is achieved through diligence with follow-up care, diagnostic Pediatric Assessment Measure (PRAM) $[8,9]$, compliance with physician-ordered medications, avoidance of triggers, and strict adherence to the nationally accepted asthma action treatment plan that would be prescribed by the healthcare provider. Disease and age-specific education for asthma will bring awareness to the patient and caregiver for self-management and self-recognition of symptoms that encourage early treatment and prevention of exacerbation of their chronic condition before an exacerbation creates a new situation (NHLBI, 2014b). Attending and participating in disease and age-specific asthma education programs has been found to improve the patients' self-management skills, improve understanding of the pathophysiology of their disease process, and bring earlier recognition and awareness of their level of control. By patients maintaining control of their asthma condition, there will be a significant decrease in the overall cost of chronic care and an improvement in outcomes and quality of life [10-13].

\section{Socioeconomic Factors Affecting Asthma Control}

Socioeconomic issues have been found to have a profound effect on asthma control. Low-income families, especially amongst African American children, have a disproportionate incidence of morbidity and mortality $[13,14]$. The substandard housing, most notably older buildings containing asbestos and dust, homes with mold, cockroach and rodent infestations, can also create an allergic environment that causes an inflammatory response within the soft tissues of the airways. This response to environmental irritants will ultimately result in an increase in the incidence of asthma, asthma flare-ups, and poor control of asthma symptoms (Environmental Protection Agency [EPA], 2016a). Family dynamics that include parents or single parents who may need to work away from home, patients with at least one family member with asthma and maternal employment outside of the home all have a direct effect on control and compliance within the adolescent patient populations due to the lack of parental supervision [15]. Poverty, a lack of transportation and living in underserved or limited access to affordable care areas, can create disparities associated with the incidence of asthma and poor asthma control. Asthma patients living at the poverty level were also found to have a higher level of emergency room visits relative to middle- and upper-income groups, denoting a subordinate level of disease control [16].

This adversity in the delivery of affordable, available healthcare, contributes to the lack of control for patients' asthma symptoms and can be directly attributed to disparities brought about by the lack of reasonable access and affordability of follow-up management of the disease. Barriers to care, perceived and real, can also be problematic in controlling asthma. Parents of children living in more impoverished environments may not have the financial wherewithal to buy needed medicine, have transportation to and from appointments, or be able to afford healthy, nutritious produce to eat and maintain a healthy weight and stave off respiratory infections, a known trigger of asthma. Financial barriers also prevent children with asthma from receiving continuous, quality healthcare that is needed to manage asthma and other chronic health conditions. Children from low-income families, typically, have a higher incidence of not only asthma but other health issues and morbidities which contributes to higher short-term and long-term health care costs [17-20]. Likewise, these health challenges can lead to lost work time for parents, lost educational opportunities for students, and may contribute to educational remediation, or family relocation, and additional failure to maintain the child's asthma.

\section{Follow up Care}

Maintaining and attending follow-up healthcare appointments, coupled with proper age- and disease-specific education, is paramount to preventing and managing asthma exacerbations, asthma flare-ups, hospitalizations, an increased use of oral corticosteroids, and deaths from uncontrolled asthma symptoms (AAFA, 2015; U.S. Department of Health and Human Services, National Heart, Lung, and Blood Institute (2014). Moreover, numerous studies in the adult and pediatric patient population have shown that an improvement in health literacy and access to affordable, available healthcare can improve overall societal health, decrease healthcare costs, and improve patient outcomes $[14,15]$. Other studies have also concluded that no-show rates for healthcare appointments typically average 25-35\% nationwide within various healthcare disciplines $[16,17]$. These underutilized, patient appointment slots have further shown to prevent or block access to care for those needing follow-up evaluations, and likely increase the time to diagnose and treat, causing unneeded pain and suffering. Ultimately, this has a negative effect for the treatment and prevention of chronic health conditions and contributes to the insurmountable financial pressure on achieving a healthier society on an already overburdened, unaffordable, and unsustainable U.S. healthcare system [18-23]. 


\section{Financial Cost of Asthma}

Medical related costs for asthma, in 2015 , exceeded $\$ 56$ billion in the U.S., including direct medical expenditures, lost time from work, including wages and productivity (AAFA, 2015). According to AAFA (2015), Asthma is the number one reason for missed days of school and loss of learning opportunities. Uncontrolled asthma is responsible for an increase in emergency room visits for uncontrolled symptoms and lack of control, hospitalizations, and admission to intensive care units. Moreover, annually, asthma is responsible for 439,000 hospitalizations, 14.2 million office visits, and 2 million emergency room visits (AAFA, 2015; CMS, 2016). Indirect and direct costs of asthma can also be attributed to noncompliance with medications and lack of follow-up care with a patient's primary care physician or pulmonologist. According to the AAFA (2015), as of 2015, $54.9 \%$ of adults and $78.3 \%$ of children were not compliant with daily asthma controller medications. This non-compliance concern adds directly to missed days from school and work, higher emergency room utilization, an increase in the use of oral steroids, and more inadequate control of the patient's asthma condition. Ultimately, this will lead to a decrease in a patient's quality of life, increasing the financial burden on the U.S. healthcare system, an increase in unneeded pain and suffering, and an increase in preventable mortality and morbidity (AAFA, 2015).

\section{Problematic No-Show Rates}

Chronic no-show rates in any healthcare discipline can have a devastating effect on the healthcare providers' ability to diagnose, treat, and manage healthcare conditions before they become a chronic health issue $[24,25]$. No-show rates also have an untoward effect on the timely access to see a physician, the length of time to begin disease-specific treatment, and the efficiency and effectiveness of the healthcare being delivered [26]. No-show rates can also have an overall negative effect on patient and physician satisfaction [27]. Non-compliance with follow-up healthcare also hurts patient outcomes as it is directly related to a failure of compliance with the corrective action or specific medical treatments that were prescribed. This failure of compliance leads to a varying degree of disease control and is, in part, due to the lack of follow-up care assessment of the initial ordered plan of action by the prescribing caregiver. When a patient does not follow-up with their provider a loss of treatment assessment and evaluation of outcomes of the disease-specific improvement plan is realized. The physician required follow-up care would allow for needed adjustment or redirection of therapy, promptly, significantly improving control or prevention of chronic disease. This disruption in care is usually a direct result of the patient or caregiver not returning for follow-up appointments [28-30].

No-show rates create an underutilization of physician or provider resources and promote non-compliance with directed physician or healthcare provider's plan of action. However, they also serve as a quality indicator as this creates a perceived reduction of cohesiveness between the patient and the primary care physician or specialty provider of care. Moreover, financial losses brought about by no-show appointments to a facility can interrupt the availability of care for others waiting to be seen, thus affecting the health of others [31-34]. No-shows rates also have an untoward effect on the patient and caregiver satisfaction, decrease specialty healthcare accessibility, create a waste of healthcare staff and facility resources, and ultimately, disrupt the management of chronic illness and diseases. As a result, the healthcare facility may be unable to withstand the losses incurred by the no-show patient and may be unable to continue to provide needed follow-up healthcare services. The resulting no-show rates further limit the expansion of services in underserved areas and create a healthcare vacuum of individuals utilizing emergency room services as a primary care modality, most notably in the pediatric asthma patient populations. This overutilization of emergency room services for preventable asthma exacerbations can overwhelm hospitals, have a significant impact in financial burdens to healthcare expenditures and block access to true emergency room patients [35].

\section{Asthma and No-Show}

Missed follow-up appointments, or patients not showing up for scheduled appointments, can have a detrimental effect in the delivery of healthcare in the U.S. No-show rates result in financial loses to physicians and healthcare facilities and cause a decrease in the efficiency and effectiveness of delivered healthcare services plus the pain and suffering of others waiting for healthcare intervention. This not only monetarily discourages individuals from pursuing a physician specialist vocation but puts a financial strain on healthcare facilities preventing the expansion of services or maintenance of present services, thereby having an untoward effect on access to care and societal health. High levels of no-show patients also create a vacuum effect of healthcare resources and create a delay in initiation of healthcare needed to prevent and manage asthma and other chronic disease and conditions [36,37]. No-show rates vary from area to area and across different health disciplines. According to Kheirkhah et al. (2015), several studies performed in a community health setting, revealed that no-show rates could be as low as $5 \%$ in some areas of healthcare, and some rates as high as $80 \%$, with the highest no-show rates, realized in subspecialty care. The average loss per appointment averaged \$196.

This financial loss was compounded by an average of 62 noshow appointments, per day, with an annual loss of $\$ 3$ million in lost revenue and patient care hours [38] Another study reported 25\% no-show rates, with another $31 \%$ of the patient population arriving late. Within this study of the extensive family practice of 45,000 patients seen, per year, the average cost of missed appointments could exceed \$ 3.2 million in lost revenue and underutilized patient appointment slots. This represents a loss percentage of $3 \%$ to $14 \%$, respectively [38]. There is also a considerable cost associated with missed appointments, not only to individuals waiting for care but 
on society overall in the form of wasted resources. Costs may also be incurred due to a missed diagnosis or a delay in treatment as the condition may develop into a more serious chronic health care condition that will require more significant healthcare resources to treat and maintain [38]. This not only results in an increase in pain and suffering for individuals waiting for care but puts an insurmountable financial strain on an already unsustainable healthcare system (Social Security Advisory Board, 2009).

A recent study conducted by Grant, Bowen, Neidell, Prinz, and Redlener revealed that, within the pediatric patient population suffering from asthma, implementation of best practices, with integration of evidence-based medicine practices, yielded an appreciable savings to the healthcare system and a decrease in flair ups, emergency room visits, hospitalizations, and overall health of the asthmatic patient [39]. Pediatric patients, ages 36 months to 19 years $(n=244)$ and follow-up $(n=202)$ were evaluated after implementation of advanced education and enhanced treatments in primary care. The subjects then followed up with strict management and care. This was then evaluated for overall costs associated with the management of their asthma condition. The savings attributed to the overall costs of managing each patient was reduced by $\$ 4,525$ per patient, or $\$ 4.2$ million in annual savings overall [40]. Asthma is one of the most common, chronic healthcare conditions afflicting children, for which there is no known cure (NHLBI, 2014b). Diligence with scheduled follow-up appointments for disease management and control and compliance with daily controller medications coupled with evidence-based physician practices is paramount in controlling the morbidity and mortality associated with patient and caregiver non-compliance [41]. Specific patient and caregiver education have been shown to affect decreasing no-show rates but is not widely performed in an age- and disease-specific manner uniformly in outpatient facilities [42]. Education can provide patients and families with information about asthma, therefore expanding their understanding of the importance of health maintenance.

Therefore, education can also mitigate medical disparities by helping patients to comprehend and perform needed medical procedures such as proper inhaler techniques and medication selection for self-management of symptoms for their chronic asthma condition [42-48]. The review of the literature revealed only a minuscule amount of existing research in ascertaining information related to the improvement in no-show rates and an overall improvement in access to asthma follow-up care achieved through asthma education. Moreover, the search for existing research about the pediatric asthma patient populations further revealed a lack of coordinated cohort studies, comparing return rates for follow-up care and pediatric asthma patients receiving age- and disease-specific asthma education. Moderator variables comparing patient demographics, such as references to participant age, gender, ethnicity, education level, financial status, geographic location, co-morbidities, such as obesity, or type of healthcare coverage, may explain the relationship [49]. Considering the scarcity of available research, more investigations examining the moderators above can provide beneficial information to support the overall health and welfare of infants, children, and adolescent asthma patient populations.

\section{Methods}

The focus of this study was to ascertain if age- and diseasespecific education affect improving the no show rates or the healthcare phenomenon of the empty chair, for follow up healthcare management in the adolescent patient asthma populations. A decrease in no show rates could conceivably affect access to those waiting for care. By improving patient appointment slot utilization, improving disease management and outcomes, this should result in an improvement of overall societal health and decreasing healthcare costs.

The following research questions were instrumental in guiding the analysis of the collected data:

1. What impact do age- and disease-specific education have on no show rates for follow up pediatric asthma management? More specifically,

a) What is the impact on appointment slot utilization: measurable by a decrease in individual no show rates for follow up asthma care?

2. If there is a measurable impact, are there specific moderators evident for the impact of education on NO SHOWS?

a) If no effect is found, is there an association of education to KEPT appointments?

3. Do demographic moderator variables such as age, gender, ethnicity, financial status or type of healthcare coverage or geographic location, have an association with NO SHOW rates.

\section{Research Design}

The research method for this study involved a quasiexperimental research design through a retrospective data review, commonly referred to as a retrospective chart review (RCR) or medical record review (MRR) of existing patient records utilizing the electronic medical records (EMR) for retrieval of pertinent medical data pertaining to no-show rates and the educational discharge process.

\section{Participants}

Participants were based on a random sample of males and females between the ages of eight to eighteen, with a diagnosis of asthma, and of those patients seen in the last five years who had not shown up for at least two consecutive appointments in one year. Unique to Ohio, minors must be accompanied by a parent, guardian, or approved caregiver. This random sample was derived within a multi-county health care facility and from multiple health-care providers. Participation in the study included all ethnicities and 
racial groups in the existing patient database. Inclusion criteria for this sample of participants included:

a) Participants who had a documented problem with not showing for follow-up care using the following criteria

b) Participant was an allergy patient, with a specific diagnosis of asthma

c) Participant or families who refused disease- and agespecific education.

This parameter was added to ascertain if this patient population returned for follow-up care of their asthma condition, as directed by their physician, or may have received prior education about their asthma condition. Prior education could have been through the community asthma outreach center, or through their primary care, as both processes are well documented within the patient's EMR. Participants were excluded from the data extraction if participants had multiple health comorbidities (such as cardiac insufficiencies, metabolic or psychological health issues) or for not having the cognitive function to understand the educational process (such as individuals with traumatic brain injury) or if they did not have a history of no-show rates.

\section{Instrumentation: Data}

Participants for this study were selected with the specific intent to evaluate the available EMR database within the pediatric pulmonary, allergy, and immunology outpatient clinic facility including only pediatric subjects who had a specific diagnosis of asthma. The specific parameters for no-show rates were set at patients that had missed or no showed for two consecutive appointments in a rolling year and did not change or call to cancel their appointment before not showing up for evaluation of their chronic asthma condition in the scheduled time slot.

\section{Procedures}

The research procedure for this study began with a request of information from the clinical supervising personal from the EMR system known as Epic (software used for maintaining medical records). Collaborative Institutional Training Initiative (CITI), including Health Insurance Portability and Accountability Act (HIPAA) education, had been completed along with the approval of both IRB proposals from collaborating institutions had been received before the informational request. The EMR search was limited to subjects within the department of pediatric pulmonary medicine, including both specific pulmonary and pediatric allergy disciplines of care. Individuals were then randomly chosen based on the inclusion and exclusion criteria set forth within this study until $n=250$ subjects were identified. Randomly chosen subjects were then placed into a category of disease- and age-specific asthma education received, or not received, and compared with when the education was received. Typical asthma education within this facility was comprised of age- and disease-specific process that included the patient, the patient's family, and any designated care provider. Participants receiving education also had a learning assessment evaluation, as well. Strict asthma guidelines, including a nationally recognized asthma action plan and school asthma action plan, were introduced to the patient along with diseasespecific education about the participant's asthma condition was provided as well.

Moreover, participants within the asthma education process had to demonstrate proficiency with inhaled medications through a visual feedback mechanism with the educator, along with disease symptom recognition as well. This provided for proficiency in early self-management. Asthma educators were also required to be proficient within the arena of asthma and disease management. The subjects were analyzed to ascertain if, after the completion of the educational process, the next appointment was completed and the missed appointments, or no-show rates, had declined. The subjects were also compared for compliance with followup appointments with those subjects who did not receive, or refused, age- and disease-specific education at the time of their appointment, or by other means through the community outreach asthma education center located near the outpatient facility. This was also documented in the patients' EMR.

\section{Results}

\section{Demographics}

A random sample of data included participants from a population within Mahoning, Trumbull, Stark and Franklin counties in Ohio with a specific ICD-9 or ICD-10 code for varying degrees of asthma diagnosis or hyperactive airway disease. Participants within the study yielded an $n=193$ with a gender distribution of 101 males or (52\%) and 92 females or (47.7\%). The racial distribution of the participant populations revealed that 90 or $46.6 \%$ were black, 63 participants or $32.6 \%$ of the participant distribution was White, 10 or $5.2 \%$ were Hispanic/Latino, and $15.5 \%$ of the participants were listed as other. Those participants that received age- and diseasespecific education included $87.6 \%$ of the participant population with $12.4 \%$ of the participant population that chose not to take part or did not receive the educational encounter for other reasons. The analysis examined the type of insurance coverage as a type of financial indicator. Medicaid Medical is a straight Medicaid type of government supplied medical coverage for those individuals who are $100 \%$ Medicaid dependent. Medicaid/Medical Care Organization (MCO) included those participants that were covered under a government assisted Health Maintenance Organization (HMO). Commercial insurance is insurance that individuals have through their employer with some individual monthly contribution. The sample of participants for this investigation included primarily Medicaid MCO (86\%), followed by Commercial (8.8\%), followed by Medicaid Medical (5.2\%).

Descriptive analytics were used to examine the following variables, including, appointments that were KEPT and the number of NO-SHOW appointments. This was represented as the 
total number of appointments for the individual. Data were also analyzed for individual appointments that were canceled and those appointments that were rescheduled to a later date before the original appointment date. This analysis is provided in Table As indicated in Table 1, KEPT appointments were highest on average, but also presented the most significant variability in the data. The values of skewness and kurtosis are within the acceptable ranges of $|2.0|$ and $|5.0|$, respectively (Field, 2009). Research question \#1 asks: What impact do age- and disease-specific education have on no show rates for follow up pediatric asthma management? More specifically, what is the impact on appointment slot utilization: measurable by a decrease in individual no show rates for follow up asthma care? An independent samples t-test was conducted on the frequency of NO SHOWS by education group. The Levene's Test for Equality of Variance revealed that homogeneity of variance was not tenable, $F(1,190)=5.32, p=.022$. The lack of homogeneity of variance across the two groups is likely due to sampling size differences across the groups. To confidently analyze this data, the degrees of freedom were adjusted downward before conducting the $t$ analysis (Field, 2009). Results indicate that there are no significant differences across the two groups, regarding NO SHOWS, $\mathrm{t}(44.78)=-1.78, \mathrm{p}=.082$.

Table 1: Descriptive statistics for variables of continuous measurement.

\begin{tabular}{|c|c|c|c|c|}
\hline & Mean & Std. Deviation & Skewness & Kurtosis \\
\hline KEPT & 12.81 & 11.13 & 1.23 & 1.06 \\
\hline No Show & 3.89 & 3.85 & 1.44 & 2.01 \\
\hline Canceled & 2.33 & 2.45 & 1.61 & 2.77 \\
\hline Rescheduled & 4.89 & 4.82 & 1.52 & 2.65 \\
\hline
\end{tabular}

Research Question \#2 asks if there is a measurable impact, are there specific moderators evident for the impact of education on NO SHOW; If no effect is found, is there an association of education to KEPT appointments? No effect was discovered based on the analysis conducted for research question \#1. Pearson's Zero-Order Correlation was conducted to assess the relationship between the education variable and the Slot Utilization Variables. These results are presented in Table 2. As indicated above, KEPT appointments are the only variable that was correlated to the education variable. Since Education and KEPT appointments are significantly correlated, it was determined that a Binary Logistic Regression would be used to assess whether the number of KEPT appointments was an indicator of the person receiving education about their condition and treatment (Field, 2009). Gender and race were added to the binary logistic regression to examine if they added to model precision. Binary logistic regression determines the impact of multiple independent variables simultaneously to predict or indicate membership of one of two dependent variable levels [50-52].
Table 2: Pearson's Zero - Order Correlation Between Education and Slot Utilization $(\mathrm{n}=193)$.

\begin{tabular}{|c|c|c|c|}
\hline Utilization & $\mathbf{R}$ & Lower $\mathbf{C I}_{\mathbf{9 5}}$ & Upper $_{\mathbf{1 9 5}}$ \\
\hline KEPT & $.175^{*}$ & .032 & .306 \\
\hline No Show & .089 & -.053 & .227 \\
\hline Canceled & .134 & -.007 & .270 \\
\hline Rescheduled & .118 & -.024 & .255 \\
\hline
\end{tabular}

Specifically, this analysis was used to assess if a patientreceived education could be estimated based on their KEPT appointments, gender, and race. The variables in the Equation Table (Table 3) have several essential elements. Results indicate a Nagelkerke R Square R2 = .122 with a 2-Log Likelihood of 132.06. This indicates that a weak but significant relationship of $12.2 \%$ exists between the predictors and the dependent dichotomous variable, Education. The Hosmer and Lemeshow test $(\mathrm{H}-\mathrm{L})$ is a test of sampling adequacy (Field, 2009). The H-L Statistic $\chi^{2} 8=10.24, p$ $=.249$, which is not statistically significant, indicating a good model fit. As indicated above, this significant model can demonstrate the likelihood of a patient receiving education, by the frequency of their KEPT appointments, above what would be predicted by chance. Research Question \#3 asks: Do demographic moderator variables such as age, gender, race, financial status/healthcare coverage or geographic zone have an association with no show rates? Pearson's Zero-order Correlation was conducted to asses if any association existed between NO SHOW rates and age, gender, ethnicity, and type of healthcare coverage (financial status).

Table 3: Variables in the equation table.

\begin{tabular}{|c|c|c|c|c|c|c|}
\hline & B & S.E. & Wald & df & Sig. & $\operatorname{Exp(B)}$ \\
\hline Constant & 0.869 & 0.592 & 2.153 & 1 & 0.142 & 2.38 \\
\hline KEPT & 0.074 & 0.031 & 5.824 & 1 & 0.016 & 1.077 \\
\hline Gender & -0.209 & 0.46 & 0.206 & 1 & 0.650 & 0.812 \\
\hline RACE & & & 4.989 & 3 & 0.173 & \\
\hline
\end{tabular}

Results indicate that there are no significant correlations found between the NO SHOW rates and the potential moderators of age, gender, ethnicity, and type of health care coverage. An additional analysis was conducted to examine if the residential location was an indicator of NO-SHOW rates and KEPT appointments. A Pearson's zero-order correlation was conducted to access if there were more missed appointments by county of the participants, the education variable, and the NO SHOW as well as KEPT appointment rates. The results are presented in Table 4. As indicated in Table 4., County is significantly correlated to the Education Variable $(\mathrm{r}=$ .173, CI95[.033, .307]). A Pearson's Chi-Square indicates that there is a significant association between County and Education, $\chi^{2} 3=$ $13.00, \mathrm{p}=.005$. 
Table 4: County by Education, NO SHOW and KEPT Appointments.

\begin{tabular}{|c|c|c|c|c|}
\hline & Education & County & KEPT & \# No Show \\
\hline Education & - & $.173^{*}$ & $.172^{*}$ & 0.089 \\
\hline County & - & & 0.082 & 0.037 \\
\hline KEPT & - & & & $.505^{* *}$ \\
\hline No Show & - & & & \\
\hline \multicolumn{5}{|c|}{${ }^{*}$ Correlation is significant at the 0.05 level (2-tailed). } \\
\hline \multicolumn{4}{|c|}{${ }^{* *}$ Correlation is significant at the 0.01 level (2-tailed). } \\
\hline
\end{tabular}

\section{Discussion and Conclusion}

This investigation intended to find if age and asthma education affected decreasing the phenomena of the "empty chair" or "no-show" rates and improving access to follow-up healthcare management in the adolescent, asthma patient populations [50]. In reducing no-show rates and improving access to follow-up care, it is conceivable that an improvement in disease management will improve overall societal health, improve quality of life, decrease suffering and the societal healthcare costs of asthma management [48]. Moreover, by decreasing no-show rates, appointment slot utilization will be improved for those waiting for a diagnosis, treatment, and follow-up management as well. Like many issues, societal, social, and cultural, with perceived moreover, real barriers may influence the problematic issue of no-show rates, this first investigation is intended as a catalyst for further studies to be performed and looks to focus on the issue of health literacy and the improvement of, or compliance with, follow-up chronic disease management. An extensive review of the literature revealed that, although many studies have been performed to understand missed appointments, or the empty chair phenomena, for follow-up disease management, a dearth of research studies have focused explicitly on the pediatric asthma patient populations. This is the first known study that employs random sampling to investigate specifically the empty chair phenomena with pediatric asthma patients.

Although commonalities existed between those receiving age- and disease-specific education and those that did not receive the educational encounter, the results of the analysis revealed that there were no significant differences across the two groups regarding no-show rates for follow-up asthma care and disease management. This is in direct contrast with the extant literature, as there are disparities within all aspects of asthma and chronic disease management between races of individuals, that include the incidence of disease, and the focus on patient education. African American populations and those living at the poverty level, most notably Hispanic /Latino populations, are two- times more likely than non-Whites to have asthma (nhlbi.gov). This may lead one to assume that as the incidence of asthma and chronic disease within a race increases, the level of no-show rates would increase for that race as well; this does not seem to be the case for those individuals who have chronic asthma within this current investigation. Interestingly, when these data were further analyzed using a Pearson's Zero-order correlation to assess the relationship between education and slot utilization variables such as KEPT, noshow, canceled and rescheduled appointment types, this analysis revealed that the KEPT appointment variable was the only slot utilization variable that correlated to the education variable.

While this result was surprising, as the focus of the investigation is on no-show rates, it is intuitive that receiving age-appropriate education should impact whether or not the patients follow their treatment plan and attend scheduled appointments. A Binary Logistical regression was conducted with the KEPT variable and revealed that the KEPT appointments variable is a significant indicator of the patient receiving an education. This analysis shows promise, as seemingly, an improvement in health literacy may have helped improve the self-awareness of the patient's (and the patient's family) chronic condition. This may be a result of the age- and disease-specific educational process as discussed within the existing literature. Health literacy, or the level at which one can process and understand basic health information, is paramount in making sound medical or life and death decisions (Tieu et al.).

As Tieu et al. posited, minorities may not have the technological wherewithal, or they may have technological disparities, such as access to a computer, the Internet or a level of technical education and financial wherewithal to afford the higher degree of technology, putting them at a disadvantage. The results suggest that with a proper level of education, there is an improvement in KEPT appointments that may allow a level of improved care for those minorities that could be technologically disadvantaged. Finally, this research investigation was an attempt to ascertain if age- and disease-specific education had an effect on improving no-show rates and if demographic variables such as age, gender, race, type of healthcare coverage, and geographic zone had a correlation to missed appointments. Pearson's Zero-order correlation was conducted to assess the association of potential moderators and the slot utilization variables.

This analysis revealed that no correlation existed between noshow rates and the previous demographic variables of age, gender, race, financial status, and county of residence. The findings of this analysis of data are in stark contrast with extant literature. According to DuMontier (2013), a cohort study revealed that individuals living within underserved and deprived areas were three times more likely to be a no-show individual for follow-up healthcare management than those that did not live in impoverished areas. Individuals who were receiving government assistance for healthcare such as straight Medicaid and Medicaid HMO were also found to be three times more likely to be a no-show individual than those individuals that were not receiving government healthcare assistance. For further investigation of the association between noshow rates and geographic zone, the analysis was utilized to further map the residential areas of the participant data by county. 
KEPT appointments were significantly different across the different county of residences represented. The highest number of no-show rates were found between Trumbull and Mahoning Counties, Ohio. These counties may need to be an area of investigation in further studies to ascertain reasons for the higher level of no-show rates in these two areas. It has been found that environmental concerns such as poor-quality housing, asbestos, smog, and ambient air pollutions all play a role in a higher incidence, and lack of, asthma control, in urban neighborhoods. This is posited to occur from the inflammatory, contributing factors associated with air pollutants and poor air quality in the urban areas of many cities. It is unclear if these areas are considered to be impoverished or low socio-economic communities, but these areas are considered to have adequate access to healthcare and are not underserved areas.

The current investigation between age- and disease-specific education delivery and a comparison to no-show rates were developed as an initial study into one of the many myriads of social and economic factors, including those of perceived and real barriers, that affect patient follow-up care. The analysis of data was an attempt to ascertain any causal influence for the problematic issue of missed healthcare follow-up appointments, with a random sample of pre-existing chart data. Moreover, while the current investigation did not reveal a significant impact of education, the prevalence of no-show rates effectively creates health concerns for others, as not showing up for appointment blocks an appointment slot for those waiting to be scheduled to be seen. This extended wait time prolongs pain and suffering, extends diagnosis and time to treatment, and invariably decreases the quality of life for those waiting to be seen. The problematic no-shows also create an issue of poor slot utilization for healthcare providers by creating underutilization of appointment slots, lost revenue opportunities, and a waste of invaluable healthcare resources. No-show rates can be best described as a societal healthcare issue that is creating burdens to the already unhealthy, unaffordable, unsustainable United States healthcare system.

\section{Limitations}

Several limitations became evident after this research study. The limitations of the data will be discussed first, followed by the limitation of the study design. The population of the data was a small randomly selected representation, $n=193$, of pediatric asthma patients in Mahoning, Trumbull, Stark, and Franklin Counties in the State of Ohio. Although significant results were found, further investigations are warranted to include a broader geographic patient population base to add power to any further investigations. However, while the sample size is limited, the sample was produced through random selection, which increases the external validity (i.e., the generalizability) of the results of the investigation's findings.

\section{Implications of this study}

The current investigation demonstrated that with education, it was $12.2 \%$ more likely that patients KEPT their appointments. These results potentially have implications for patient satisfaction, outcomes, and quality of life, and timely follow-up asthma care. Healthcare facilities that treat pediatric asthma patients will also benefit from a decrease in no-show rates, as there is a financial deficit that increases with the progression of continued daily no-show rates. Facilities that schedule appointment slots rely on revenues generated by the patient-provider encounter to expand services and provide care for a larger patient population. With a high no-show rate, a facility may have to limit services or available appointments, restricting care to those in need. Future investigation may allow an analysis of health literacy, or barriers, real and perceived, that may be causation for the higher no-show rates. By addressing the areas mentioned above of concern, an overall improvement in healthcare delivery within these areas may be realized. If physician ordered therapy is to be effective, uninterrupted management of one's chronic condition must be maintained for an improvement in overall healthcare to be realized.

\section{Conclusion}

The results are surprising in that there was no correlation found between the no-show rates and the demographic variables of age, gender, race, and type of healthcare coverage, along with no difference between groups. However, the KEPT appointment variable and county of residence were both associated with receiving age- and disease-specific education. The highest KEPT appointments were found in Mahoning County, with the highest number of no-shows found on the border of Mahoning and Trumbull Counties, Ohio. To fully understand causation for the problematic no-show issues for follow-up asthma management will indeed require further investigation. This investigation demonstrated that education might have an integral relationship with the slot utilization variable of KEPT appointments. As such, KEPT appointments seems a likely focus of further research in improving slot utilization. Ultimately the goal of this research is to understand causation for problematic no-show rates, and decreasing their overall influence on healthcare delivery, like this, improving access to care for those waiting to be seen, diagnosed, and treated for their chronic asthma condition.

Age- and disease-specific education invoke responsiveness to an improvement in health literacy through specific educational encounters that will foster improvement in KEPT appointments, therefore, improving appointment slot utilization and protecting vulnerable needed healthcare resources. This improvement in slot utilization will have far-reaching implications as it will not only improve the quality and access of healthcare delivery in the pediatric asthma patient populations, show an improvement 
in patient outcomes, and protect the integrity of the healthcare delivery process, but will ultimately make a difference in the quality of the life of a child.

\section{References}

1. Adler L, Hoagland GW (2012) Bipartisan Policy Center, What is driving U.S. healthcarespending? America's unsustainable health care cost growth 1-27.

2. Al Jahdali H, Ahmed A, Harbi A, Khan M, Salih S, et al. (2013) Improper inhaler technique is associated with poor asthma control and frequent emergency department visits. Allergy, Asthma \& Clinical 9(1): 8.

3. (2014) American College of Allergy, Asthma \& Immunology.

4. Arnold DH, Gebretsadik T, Hartert TV (2013) Spirometry and PRAM severity score changes during pediatric acute asthma exacerbation treatment in a pediatric emergency department. Journal of Asthma 50(2): 204-208.

5. (2015) Asthma and Allergy Foundation of America. Asthma facts and figures.

6. Baron R, Kenny D (1986) The moderator-mediator variable distinction in social psychological research: Conceptual, strategic, and statistical considerations. Journal of Personality and Social Psychology 51(6): 1173-1182.

7. Bloomberg GR, Banister C, Sterkel R, Epstein J, Bruns J, et al. (2009) Socioeconomic, family, and pediatric practice factors that affect the level of asthma control. Pediatrics 123(3): 829-835.

8. Bodenheimer T, Chen E, Bennett HD (2009) Confronting the growing burden of chronic disease: Can the U.S. health care workforce do the job? Health Affairs 28(1): 64-74.

9. Boise E (2014) Patient education and designing an asthma action plan. Otolaryngologic Clinics of North America 47(1): 127-134.

10. Celano M, Holsey C, Kobrynski L (2012) Home-based family intervention for low-income children with asthma: A randomized controlled pilot study. Journal of Family Psychology 26(2): 171-178.

11. (2015) Centers for Disease Control and Prevention. Control of asthma.

12. (2016) Centers for Disease Control and Prevention. Federal Advisory Committee.

13. (2016) Centers for Disease Control and Prevention. National Center for Health Statistics.

14. Chakraborty S, Muthuraman K, Lawley M (2013) Sequential clinical scheduling with patient no-show: The impact of pre-defined slot structures. Socio-Economic Planning Sciences 47(3): 205-219.

15. Chang J, Freed G, Prosser L, Patel I, Erickson S, et al. (2014) Comparisons of health care utilization outcomes in children with asthma enrolled in private insurance plans versus Medicaid. Journal of Pediatric Health Care 28(1): 71-90.

16. Cook D, Dixon P, Duckworth WM, Kaiser MS, Koehler K, et al. (2000) Beyond traditional statistical methods. 56(3): 230-233.

17. Delfino R, Tjoa T, Gullesserian S, Nickerson B (2014) Asthma morbidity and ambient air pollution: Effect modification by residential trafficrelated air pollution. Journal of Epidemiology 25(1): 48-57.

18. De Voe J (2008) The unsustainable United States health care system: A blueprint for change. Annals for Family Medicine 6(3): 263-266.

19. Dowell ML, Larwin KH (2013) Gender differences in the compensation, promotion track, and performance evaluations for school superintendents. Journal of Research in Gender Studies 1(3): 11-36.

20. Dreiher J, Goldbart A, Hershkovich J, Vardy DA, Cohen AD (2008) Factors associated with non-attendance at pediatric allergy clinics. Pediatric Allergy and Immunology 19(6): 559-563.
21. Du Montier C, Rindfleisch K, Pruszynski J, Frey JJ (2013) A multi-method intervention to reduce no-shows in an urban residency clinic. Family Medicine 45(9): 634-641.

22. (2016) Environmental Protection Agency. An introduction to indoor air quality.

23. (2016) Environmental Protection Agency Asthma.

24. Field A (2009) Discovering statistics using SPSS. Sage, Los Angeles, CA.

25. Gaudreau K, Stryhn H, Sanford C, Cheverie C, Conklin J, et al. (2014) Use of emergency departments and primary care visits for asthma-related conditions in the three years following an asthma education program. Journal of Asthma 51(3): 288-293.

26. Glover S, Piper C, Williams E, Bennett K, Thompso W, et al. (2012) Preventable asthma episodes among urban/rural children and adolescents: A comparative study. Journal of Health Disparities Research and Practice 5(2): 2010-2012.

27. Goldman A, Mayer J (2011) The no-show phenomenon. Springfield, MA: Baystate High Street Health Center.

28. Grant R, Bowen S, Neidell M, Prinz T, Redlener I (2010) Health care savings attributable to integrating guidelines-based asthma care in the pediatric medical home. Journal of Health Care for the Poor and Underserved, Suppl. Social Determinants of Children's Health 21(2): 8292.

29. Grineski S (2008) Coping with asthma in the central city: Parental experiences with children's health care. Journal of Health Care for the Poor and Underserved 19(1): 227-236.

30. Guzek LM, Gentry SD, Golomb MR (2015) The estimated cost of "noshows" in an academic pediatric neurology clinic. Pediatric Neurology 52(2): 198-201.

31. Kheirkhah P, Feng Q, Travis LM, Tavakoli Tabasi S, Sharafkhaneh A (2015) Prevalence, predictors, and economic consequences of noshows. Health Services Research 16(13): 1-8.

32. Mc Donough B, Mault S (2013) Non-attendance at a difficult-asthma clinic. Nursing Times 109(16): 12-14.

33. Molfenter T (2013) Reducing appointment no-shows: Going from theory to practice. Substance Use Misuse 48(9): 743-749.

34. Moorman J, Akinbami L, Bailey C, Johnson C, King M, et al. (2012) Centers for Disease Control and Prevention. National surveillance of asthma. The United States, 2001-2010. Atlanta: National Center for Health Statistics (U.S.); National Center for Environmental Health (U.S.).

35. Myers TR, Tomasio L (2011) Asthma: 2015 and beyond. Respiratory Care 56(9): 1389-1410.

36. (2016) National Institute on Drug Abuse.

37. Parikh A, Gupta K, Wilson AC, Fields K, Cosgrove NM (2010) The effectiveness of outpatient appointment reminder system in reducing no-show rates. The American Journal of Medicine 123(6): 542-548.

38. Phillip EJ, Du Hamel K, Jandorf J (2010) Evaluating the impact of an educational intervention to increase CRC screening rates in the African American community: A preliminary study. Cancer Causes Control 21(10): 1685-1691.

39. Rodriguez Pacheco R, Negro Alvarez JM, Campuzano Lopez FJ, Pellicer Orenes F, Murcia Aleman T, et al. (2007) Non-compliance with appointments amongst patients attending an allergology clinic, after implementation of an improvement plan. Allergol et Immunopathol 35(4): 136-144.

40. Samorani M, La Ganga LR (2015) Outpatient appointments scheduling given individual day-dependent no-show predictions. European Journal of Operational Research 240(1): 245-257.

41. Samuels RC, Ward VL, Melvin P, Macht Greenberg M (2015) Missed appointments: Factors contributing to high no-show rates in an urban pediatric primary care clinic. Clinical Pediatrics 54(10): 976-982. 
42. Schatz M, Rachelefsky G, Krishnan (2009) Follow-up after acute asthma episodes: What improves future outcomes? ATS Journals 6(4): 1-20.

43. Schauman O, Aschan LE, Arias N, Beards S, Clement S (2013) Interventions to increase initial appointment attendance in mental health services: A systematic review. Psychiatric Services 64(12): 1249-1258.

44. (2009) Social Security Advisory Board. The unsustainable cost of health care.

45. Tamesi GP, Covar RA, Strand M, Liu AH, Szefler SJ, et al. (2013) Predictors for asthma at age seven years for low-income children enrolled in the childhood asthma prevention study. The Journal of Pediatrics 162(3): 536-542.

46. Tuli SY, Thompson LA, Ryan KA, Srinivas GL, Young CM, et al. (2010) Improving quality and patient satisfaction in a pediatric resident continuity clinic through advanced access scheduling. Journal of Graduate Medical Education 2(2): 215-221.

47. (2014) US Department of Health and Human Services, National Heart, Lung, and Blood Institute. National asthma education and prevention program.

ISSN: 2574-1241

DOI: 10.26717/BJSTR.2019.19.003373

Kelly L Colwell. Biomed J Sci \& Tech Res

(C) This work is licensed under Creative Commons Attribution 4.0 License

Submission Link: https://biomedres.us/submit-manuscript.php
48. (2014) US Department of Health and Human Services, National Heart, Lung, and Blood Institute. What is asthma?

49. Van Baar JD, Joosten H, Car J, Freeman GK, Partridge MR, et al. (2006) Understanding the reasons for asthma outpatient (non)- attendance and exploring the role of telephone and e-consulting in facilitating access to care: Exploratory qualitative study. Quality and Safety in Health Care 15(3): 191-195.

50. Van Dieren Q Rijckmans MJN, Mathijssen JJP, Lobbestael J, Arntz AR (2013)Reducing no-show behavior at a community mental health center. Journal of Community Psychology 41(7): 844-850.

51. Vassar M, Holzman M (2013) The retrospective chart review: Important methodological considerations. Journal of Educational Evaluation for Health Professionals, PMC3853866.

52. Zorc JJ, Scarfone RJ, Li Y, Hong T, Harmelin M, et al. (2003) Scheduled follow-up after a pediatric emergency department visit for asthma: A randomized trial. Pediatrics 111(3): 495-502.

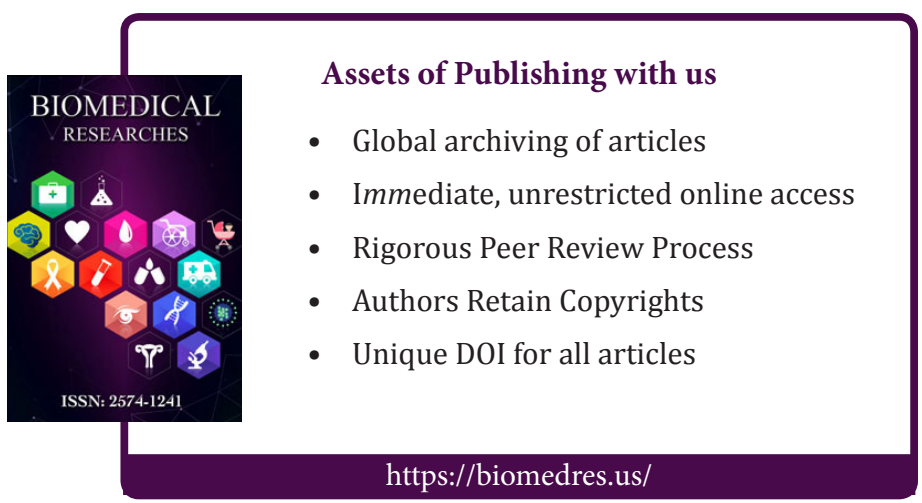

